\title{
Postprint Version
}

Martyn Whitley, Michael Newton, Glen McHale and Neil Shirtcliffe, The Self Assembly of Superhydrophobic Copper Thiolate Films on Copper in Thiol Solutions, Zeitschrift für Physikalische Chemie 226 (3) (2012) 187-200; DOI: 10.1524/zpch.2012.0140. The following article appeared in Z. Phys. Chemie and may be found at http://www.oldenbourg-link.com/doi/abs/10.1524/zpch.2012.0140.

This article may be downloaded for personal use only. Any other use requires prior permission of the author and the Oldenbourg Wissenschaftsverlag GmbH. Copyright @2012 Oldenbourg Wissenschaftsverlag $\mathrm{GmbH}$.

\section{The Self Assembly of Superhydrophobic Copper Thiolate Films on Copper in Thiol Solutions}

\author{
Martyn Whitley, Michael Newton, Glen McHale and Neil Shirtcliffe* \\ School of Science and Technology, Nottingham Trent University, Clifton Lane, Nottingham, NG11 8NS, UK \\ * Corresponding author
}

Faculty of Technology and Bionics, Hochshule Rhein-Waal, 47533 Kleve, Germany (D)

\begin{abstract}
It is common to think of thiols as molecules that form self-assembled monolayers on gold or similar metals, they have also been used as corrosion inhibitors on copper and steel, but we show here that if the copper remains in thiol solution corrosion is accelerated and a thick layer of corrosion products with a very rough surface forms. The layer is superhydrophobic; repelling water so effectively that it rolls off leaving the surface completely dry. It is composed of a copper (I) thiolate complex and growth occurs quite rapidly under normal conditions. The film morphology depends upon the solvent and thiol used. The presence of organic amines in the solution leads to the formation of thinner layers by removing corrosion products into solution, allowing the removal of native oxide layers.
\end{abstract}

Keywords Superhydrophobic; thiol; copper thiolate; thick film; oxidation; corrosion; SAM. 


\section{Introduction}

Thiols are often used to generate self-assembled monolayers (SAMs) on gold surfaces. The thiol group binds strongly to gold and similar metals. This is often used to generate functionalised metal surfaces and to protect metals from the environment or hide them from biological systems.

Thiols can be oxidized to disulfides, further oxidation to sulfonates and then to sulfates is possible; the oxidation reaction is catalyzed in solution by copper ions. Oxidation to sulfonate is known to occur in thiol SAMs at metal surfaces and to lead to dissolution of the SAM. In some cases UV mediated oxidation can be used to pattern the thiol SAMs.[1] In this case the thiol layer is exposed to UV radiation through a mask, which produces ozone that oxidizes the thiols to sulfonates and then the patterned layer is placed into a different thiol solution. The second thiol displaces the sulfonates in the patterned areas, leaving a SAM with chemical patterning[2,3].

When thiols are deposited on copper or silver the oxide at the surface of the metal can oxidize the thiol and itself be reduced to the metal. This leads to the removal of the oxide layer on the metal and to the formation of a disulfide or sulfonate that can desorb from the surface and be replaced with new thiol molecules. In this way it is often considered possible to treat copper surfaces with thiols to produce a functionalized, oxide-free, metal-organic interface. In practice some oxygen is usually left at the interface and there has been some speculation as to what form this might be in[4] and the layer seldom behaves as well as those on gold or even silver[5].

Despite this thiols have been used to protect copper surfaces against corrosion, this has been shown to be an efficient method under some circumstances as the thiol layer is insoluble in aqueous solvents and acts as a diffusion barrier for oxygen and other attacking agents. At low temperatures the thiol monolayers can protect copper against corrosion for a considerable time, at room temperature reaction is slowed but not halted as oxide grows beneath the thiol layer.[6,7]

We noticed that if copper samples are allowed to stand in a solution of alkanethiol in ethanol for a long period they become covered with a whitish layer that, once dried, repels water very effectively. The extreme contact angle and water repellency indicates that this surface is superhydrophobic and therefore can be expected to have a combination of micro- or nano-scale roughness and hydrophobicity[8].

This paper describes an investigation of the formation of this thick film on copper in thiol solutions and one way how this might be avoided.

\section{Materials and Methods}

$2 \mathrm{~cm} \times 1 \mathrm{~cm}$ rectangular copper samples were cut from copper sheet (0.25 mm 99.98\% Aldrich UK)

Oxide was cleaned off the copper rectangles by abrading them with a polymer wool pad (Scotchpad 3M). They were then sonicated in ethanol for 10 minutes, rinsed and dried before use.

Octanethiol (98\% Aldrich UK) was mixed with one of a group of selected solvents in a $15 \mathrm{ml}$ polypropylene centrifuge tube, a copper sample added and the tube closed and stored upright in a fume cupboard. Sets of samples with different solvents were prepared together to ensure similar temperature conditions. The thiol concentration was $0.576 \mathrm{M}$ for the samples shown here, although smaller concentrations also gave rise to thick films over longer periods as long as sufficient total thiol was present.

Solvents used were methanol, ethanol, isopropanol (Fisher UK) and butanol (Aldrich UK, 99\%); as supplied and with $10 \% \mathrm{v} / \mathrm{v}$ water added, toluene, ethanolamine, diethylentriamine and n-butylamine (Sigma Aldrich UK, 99.5\%, 98\%, $99 \%, 99 \%)$. 
In other experiments the same concentration of thioethanol (mercaptoethanol) (Sigma Aldrich UK 99\%) or dibutyldisulfide (Sigma Aldrich UK, 97\%) in ethanol were used to investigate the effect of sulfur oxidation state and thiol polarity.

The copper samples developed a layer of reaction materials that was investigated after 8 days using scanning electron microscopy (SEM) in a Jeol JSM 840A, contact angle measurement using a Krüss DSA10, Infrared spectroscopy using a Nikon Magna 750 and X-ray photoelectron spectroscopy (XPS) using a Vacuum Generators ESCALAB 5 Mark II, with an Al source.

A small number of copper samples were treated electrochemically in the same alcohol/thiol mixtures. Two copper strips 5 by $0.5 \mathrm{~cm}$ were inserted through a lid parallel to one another $10 \mathrm{~mm}$ apart and dipped $2 \mathrm{~cm}$ into $4 \mathrm{~mL}$ of the thiol mixture in a small vial. A D.C. power supply was connected to them and they were polarized at $15 \mathrm{~V}$ for $2 \mathrm{~h}$. In this time a superhydrophobic whitish film was formed. A methanol/octanethiol film formed in this way was compared with one formed as before by infrared spectroscopy.

\section{Results and Discussion}

\subsection{Film Structures}

As can be seen from Figure 1 the structures formed in alcohol-thiol mixtures were similar in structure to one another. The films showed a combination of small pores and long, deep cracks that appear to extend through the film. The size of the small pores increased as the alcohol used decreased in polarity. The number of cracks, on the other hand, decreased, with the least polar, butanol being almost crack free. This suggests that the cracks form during drying as liquid drying in smaller pores will exert a higher compression force on the material.

When water was added to the alsohols (Figure 2a and b) the resulting layers appeared as if they had been produced in a more polar alcohol. This is to be expected if the layer is formed in a dissolution-deposition process as the polarity of the solvent as a whole will determine the solubility. Films produced over longer times (not shown here) continued this theme with less polar alcohols giving rise to larger crystal structures, plates and tubes.

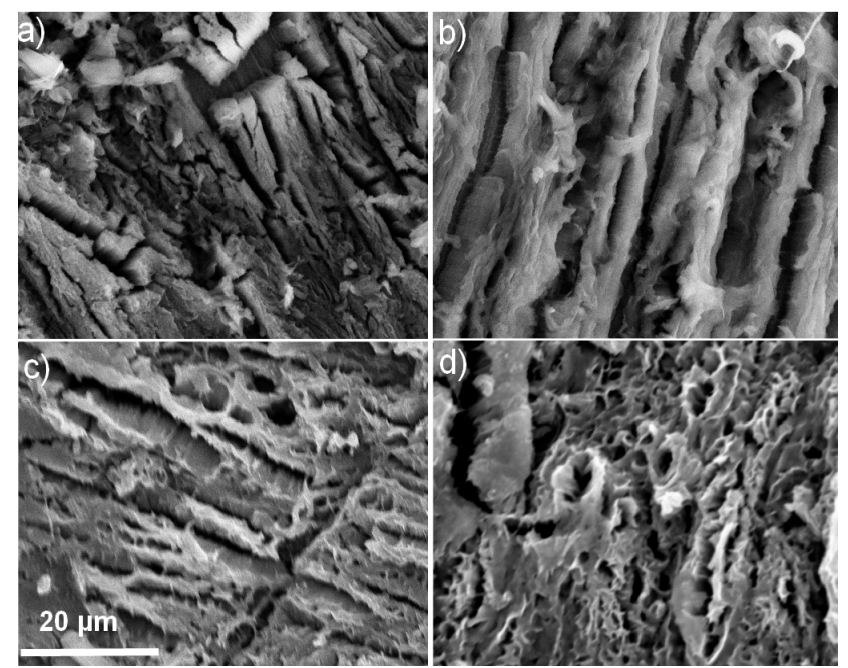

Fig. 1. SEM micrographs of layers formed on copper after 8 days using $0.576 \mathrm{M}$ octanethiol in different alcohols a) methanol b) ethanol c) isopropanol d n-butanol. 

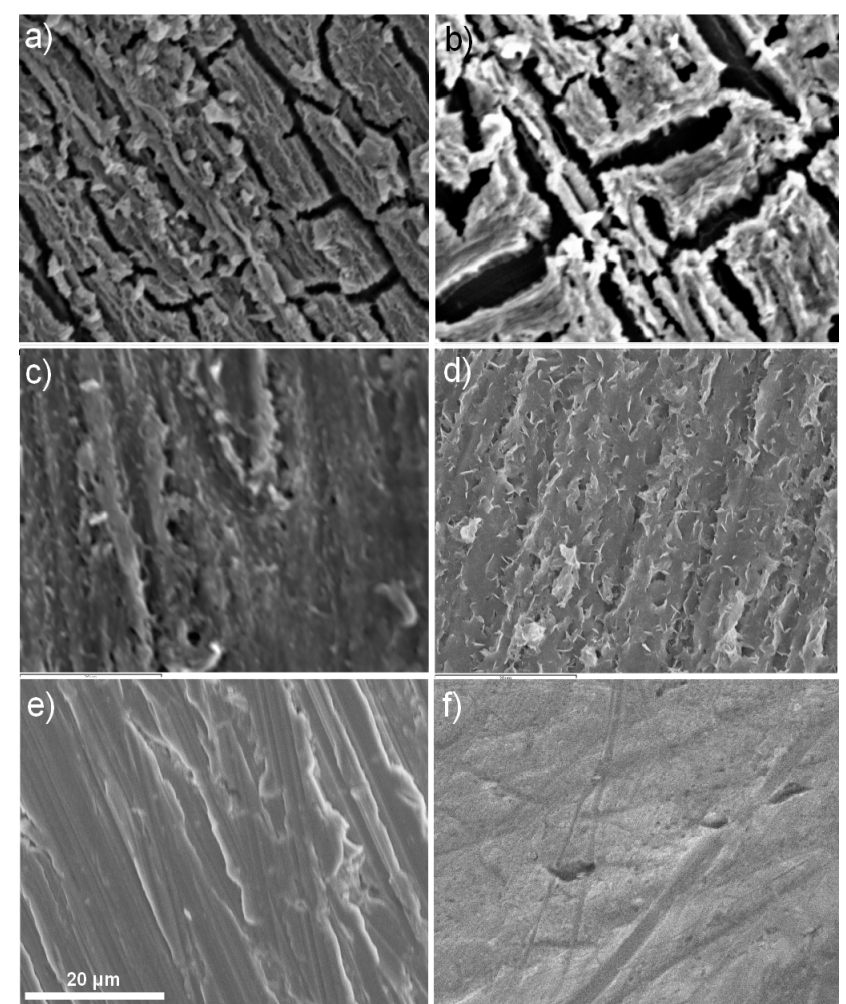

Fig. 2. SEM micrographs of layers formed on copper after 8 days using $0.576 \mathrm{M}$ octanethiol in different solvents a) ethanol/10\% water b) isopropanol/10\% water c) acetone (2 propanone) d) toluene e) reference sample before film growth $\mathrm{f}$ ) after 8 days in butylamine/octanethiol

The films produced in different alcohols were similar to one-another in thickness and structure. When the samples were flexed and then imaged in the SEM at an angle the thickness of the films could be estimated from delaminated areas. The samples grown without added water were all around $13 \mu \mathrm{m}$ thick after 8 days at room temperature. This suggests that there is a common rate-determining factor, probably solid state mass transfer or reaction at the interface between the thiol complex and the copper oxide. Addition of water reduced this thickness to around $4 \mu \mathrm{m}$ for all of the alcohols tested except methanol, where the three liquids would not mix in the desired ratio.

The aprotic solvents acetone and toluene produced much thinner layers in the same period, the structure of the layers were also considerably different from those formed in alcohols with no cracks and few pores being visible but flakes of material being evident at the surface (Figure $2 \mathrm{c}$ and $\mathrm{d}$ ). Acetone is similar in many respects to alcohols, having similar oxygen saturation concentration to butanol[ $[9,10]$ and having similar polarity (Hansen solubility parameter) to methanol[11]. This suggests that the large difference in layer growth is due to acetone's aprotic nature and its effects on the growth reaction. Reaction of the thiol with copper ions will generate protons that can react with the oxide/hydroxide. The movement of protons is facilitated by a protic solvent.

Thioethanol in ethanol also produced a visible layer but dibutyldisulfide did not. The morphology of the thioethanol film differed from that of the octanethiol, supporting the dissolution-redeposition mechanism as this thiol will form more polar compounds. The fact that disulfide (oxidized thiol) did not form a layer suggests that a redox reaction is responsible as the disulfide is the oxidised form of the thiol.

\subsection{Superhydrophobicity}

Alkanethiol films are hydrophobic, showing contact angles of around $95^{\circ}$. Superhydrophobicity arises when surface structure of a suitable size magnifies hydrophobicity to extreme values, typically $>150^{\circ}$. Under some definitions both the advancing and receding angles must be high for a surface to be defined as superhydrophobic and in this case water drops roll rapidly off the surface[8]. 
Many of the films showed very high contact angles with water and most of them were superhydrophobic, drops of water sat on them like beads (Figure 3 ) and rolled off easily.

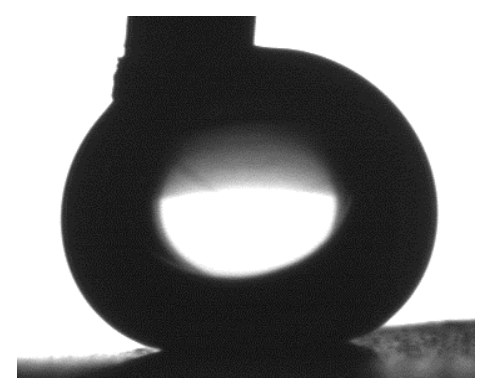

Fig. 3. Photograph of a drop of water on a thiol film formed in 2propanol

Drops of water were placed on the surfaces and their volumes increased and decreased in order to measure both advancing and receding angles. The advancing angle is a measure of how much like a sphere a drop will appear when it is deposited. The difference between the advancing (increasing volume) and receding (decreasing volume) angles is known as the contact angle hysteresis. The difference between the cosines of the advancing and receding angles is a measure of the stickiness of the surface in that it determines how likely it is that a drop on the surface can slide or roll off. As can be seen from the sliding angles presented, surfaces with low contact angle hysteresis allow drop sliding or rolling at low tilt angles and at higher hysteresis small drops adhere completely and cannot be removed by tilting (labeled as "sticks").

The advancing and receding contact angles on the alcohol-thiol layers were all similar, with all of the surfaces showing superhydrophobicity and low contact angle hysteresis except for the films formed in ethanol (Table 1). The greater hysteresis of the ethanol films was expected as the film was smoother than the others. After longer reaction periods this film became more water repellent and behaved like the others.

\begin{tabular}{|c|c|c|c|}
\hline Solvent & $\begin{array}{l}\text { Advancing } \\
\text { contact angle } \\
\text { (degrees) }\end{array}$ & $\begin{array}{l}\text { Receding } \\
\text { Contact } \\
\text { angle } \\
\text { (degrees) }\end{array}$ & $\begin{array}{l}\text { Sliding An- } \\
\text { gle } 10 \mu \mathrm{l} \\
\text { drop } \\
\text { (degrees) }\end{array}$ \\
\hline methanol & 166 & 158 & 3 \\
\hline ethanol & 155 & 109 & sticks \\
\hline 2-propanol & 162 & 159 & 1 \\
\hline butanol & 141 & 138 & 5 \\
\hline methanol_water & 166 & 159 & 2 \\
\hline ethanol_water & 147 & 146 & 2 \\
\hline 2-propanol_water & 167 & 162 & 1 \\
\hline butanol-water & 159 & 102 & sticks \\
\hline acetone & 123 & 11 & sticks \\
\hline toluene & 145 & 5 & sticks \\
\hline ethanol 1 hour & 95 & 45 & sticks \\
\hline butylamine & 95 & 25 & sticks \\
\hline $\begin{array}{l}\text { mercaptoethanol in } \\
\text { ethanol }\end{array}$ & 75 & 7 & sticks \\
\hline
\end{tabular}

Table 1. Contact angles on layers formed in different conditions, advancing angles are the largest angle measured as the volume of a drop is increased slowly, receding angles the lowest values as it is decreased. Sliding angles for $10 \mu \mathrm{L}$ drops calculated from Furmidge's equation as an example.

The different shaped surfaces observed on toluene-thiol and acetone-thiol showed high advancing contact angles with very high hysteresis. 
Water on rough hydrophobic surfaces is often assumed to be in one of two states. If the entire surface is wetted the interfacial area between liquid and solid is high so although the advancing contact angle can be large any hysteresis is also increased. If the roughness is great enough gas pockets can be retained beneath the water and the drop then sits on a mixed interface of solid and gas. This reduces interfacial contact between the water and the solid and leads to low hysteresis and rolling in extreme cases. The amount of interfacial contact determines the contact angle hysteresis and the stickiness of the surface to a great extent although there are other effects that can be important. In most cases intermediate states between the two extreme models are observed. Further information on superhydrophobicity and further references can be found in recent reviews[8].

The thiol surfaces with low hysteresis are highly porous with very deep pores and fissures and show no continuous ridges to which the contact line could stick. Those with high hysteresis, such as those produced in the aprotic solvents show long ridges and less overall roughness. This suggests that the high hysteresis of some surfaces arises from a combination of continuous features at the contact line and a higher interfacial contact area.

The structure of the film must have evolved over time as a copper surface of this type immersed into alkanethiol solution for 1 hour showed contact angles of around $95^{\circ}$, as would be expected for alkanethiols on flat gold, this agreed with SE M images of the surfaces after short times that showed only scratches from the preparation process (identical to Figure 2e).

Thick films produced from thioethanol were slightly hydrophilic, as might be expected. The very thin layers formed from dibutyldisulfide showed relatively high contact angle hysteresis, an indication that they were patchy, showing local hydrophobicity and hydrophilicity as they were not rougher than a thiol film on the same substrate.[12] As reported by Sung[13] disulfides cannot reduce the oxide so they form incomplete layers. On metallic surfaces films formed from thiols and dithiols are indistinguishable, suggesting that in the experiments here the native copper oxide is reduced by the thiol to form the thick layer.

\subsection{Film Chemistry}

\subsubsection{XPS}

X-ray photoelectron spectroscopy of the films using an ESCALAB 5 Mark II, an Al source and irradiation and takeoff angles of $45^{\circ}$ revealed that the films contain all of the elements expected, $\mathrm{Cu}, \mathrm{O}, \mathrm{C}$ and $\mathrm{S}$ (Figure 4). Indicative peaks for each element were integrated and converted into atomic percentages in the detection volume. The copper to sulfur ratio is slightly higher than 1:1 and there is a small amount of oxygen present (Table 2). In Figure 5 the survey spectrum has been labeled with the peak identities and the smaller regions the doublets have been labeled with their spin orbit numbers so that they can be considered together.

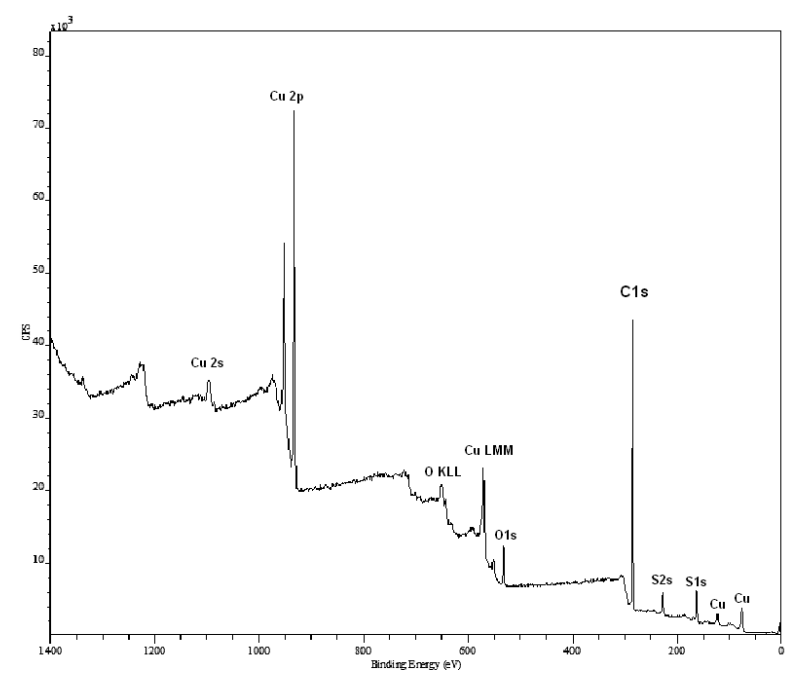

Fig. 4. XPS survey scan of a film produced in methanol 

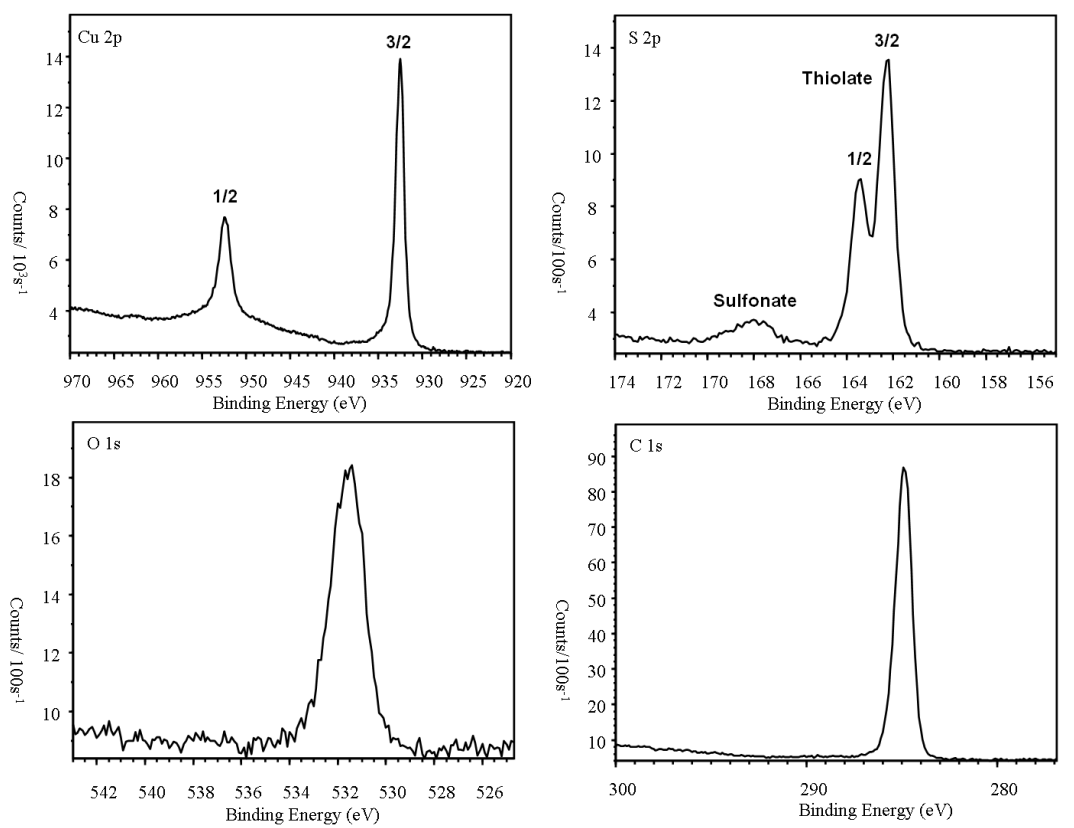

Fig. 5. XPS narrow scans of a film produced in methanol from top left $\mathrm{Cu} 2 \mathrm{p}, \mathrm{S} 2 \mathrm{p}$, $\mathrm{O} 1 \mathrm{~s}, \mathrm{C} 1 \mathrm{~s}$.

\begin{tabular}{|l|l|r|r|}
\hline $\begin{array}{l}\text { Element } \\
\text { (transition) }\end{array}$ & $\begin{array}{l}\text { Heptanethiol } \\
\text { in Methanol } \\
\text { (Atomic \%) }\end{array}$ & $\begin{array}{l}\text { Heptanethiol } \\
\text { in Acetone } \\
\text { (Atomic \%) }\end{array}$ & $\begin{array}{l}\text { Thiobutanol } \\
\text { in Methanol } \\
\text { (Atomic \%) }\end{array}$ \\
\hline $\mathrm{Cu} \mathrm{2p}$ & 9.5 & 10.6 & 10.4 \\
\hline $\mathrm{O} 1 \mathrm{~s}$ & 4.6 & 1.3 & 23.1 \\
\hline $\mathrm{C} 1 \mathrm{~s}$ & 78.8 & 81.3 & 52.1 \\
\hline $\mathrm{S} 2 \mathrm{p}$ & 7.1 & 6.8 & 14.5 \\
\hline
\end{tabular}

Table 2. Elemental Analysis from XPS broad Scans Averages of Three Areas on three different samples, values are atomic number percent calculated from peak area.

Looking at Copper $2 \mathrm{p}$ region reveals that the copper is not in the +2 state (a satellite to high binding energy is observed with $\mathrm{Cu}$ (II) and is not present here, only the spin orbit split doublet is present (Figure 5))[14]. In Cu II several shake up peaks are observed, due to electrons in the comound jumping levels at the same time the photoelectron leaves. In effect the same process that gives rise to colour occurs at the same time as the XPS process leading to extra signals. Examples and further explanation can be found in the online thesis of D. Pop[14].

The carbon signal, the C1s peak was sharp and well defined in all of the samples; it was assumed to be aliphatic carbon as this is the most prominent carbon in the sample and was fixed at $285.0 \mathrm{eV}$ to compensate peak shifts for sample charging. Fitting of the $\mathrm{C} 1 \mathrm{~s}$ peak revealed no other components except a small contribution from thiol carbon, $\mathrm{C}-\mathrm{S}$ at $287.6 \mathrm{eV}$.

The sulfur S2p peak is a doublet and the main part was observed at $162.2 \mathrm{eV}$ with its partner next to it, typical of thiols bound in a thiolate. Fitting revealed a small peak, less than $10 \%$ of the main one by area, at around $163 \mathrm{eV}$ typical of unbound thiol. This is often observed in thiol layers and is due to unreacted thiol in or on the film. The small peak (doublet) to higher binding energy $168 \mathrm{eV}$ is indicative of a sulfonate, and shows some of the thiol has become oxidised[4]. This species has been observed before on the surface of thiol films after they were exposed to the atmosphere[15]. Fitting revealed all of these to have their doublet partners and no other significant signals. As the sample was stored for several days in the air before testing it is unsurprising that a thin layer at the surface became oxidised in this time. 
This is supported by the O1s peak, which occurs at around $531.5 \mathrm{eV}$, where sulfonates $(531.8 \mathrm{eV})[16]$ and $\mathrm{Cu} 2 \mathrm{O}$ $(530.6 \mathrm{eV})$ are found and far from $\mathrm{CuO}(529.8 \mathrm{eV})[17]$.

The elemental ratio of copper and sulfur (Table 2) and the copper oxidation state of 1 suggest that the bulk of the material is made up of copper (I) thiolate and a small proportion of the thiol has been partially oxidised to the sulfonate. The excess of copper compared with sulfur and the peak positions suggests that some $\mathrm{Cu} 2 \mathrm{O}$ was present in the layers.

Although all of the peaks were fitted these results are not presented here because no additional peaks were found apart from the small unbound thiol in the $\mathrm{S} 2 \mathrm{p}$. As this material was a small contribution and not chemically bound we have concentrated on the nature of the rest of the layer.

\subsubsection{Infrared Measurements}

Reflection infrared (IRRAS) was performed on some of the samples to investigate deeper inside the films as the measurement depth of reflection IR is greater than that of XPS.

Diffuse reflection infrared measurement (surface) was compared with measurements of a powdered sample (bulk) (Figure 6). This measurement showed that there is very little hydroxide in the sample as no absorption was observed at $3500 \mathrm{~cm}^{-1}$. It also showed that there was a contribution of $\mathrm{Cu}_{2} \mathrm{O}$ at $610 \mathrm{~cm}^{-1}$ that increased when the bulk of the film was measured (ground sample instead of diffuse reflection), indicating that the film is vertically structured with (mostly) thiolate at the top and some oxide at the bottom. The small signal around $550 \mathrm{~cm}^{-1}$ could be due to a dithiolate S-S, which normally appears at $500-540 \mathrm{~cm}^{-1}$ or could be a small amount of $\mathrm{CuO}$, which has peaks at 460 and $530 \mathrm{~cm}^{-1}[18]$.

The electrochemically generated film showed very similar features over most of the spectrum but no evidence of copper oxides or dithiolate. This suggests that the electrochemically deposited film is composed of the pure copper thiolate. It is likely in this case that copper ions formed at the electrode react directly with the thiol to form the copper (I) thiolate complex and that oxygen is not involved in the reaction.

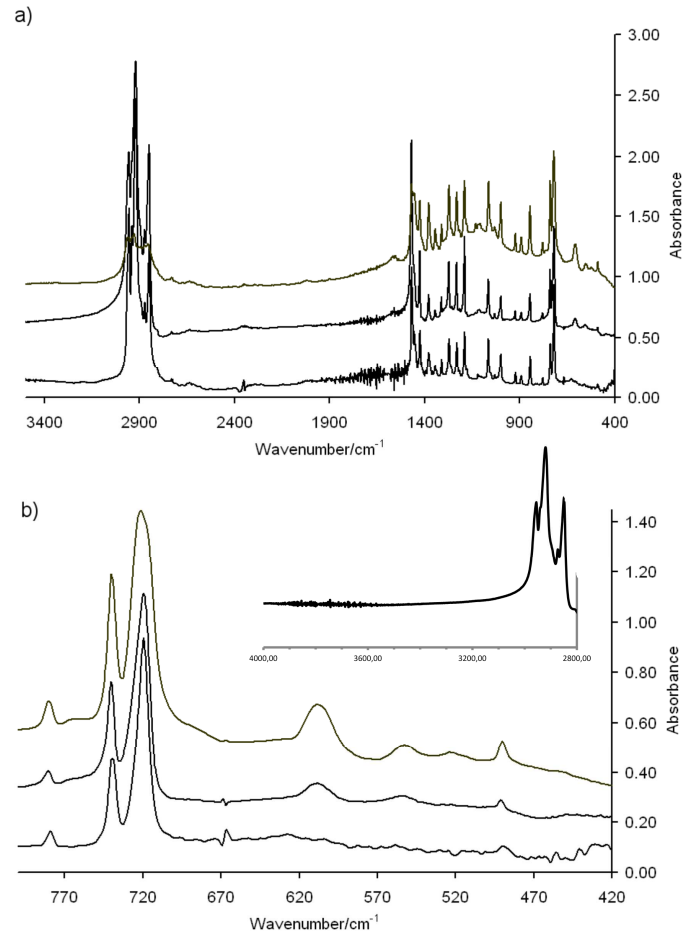

Fig. 6. Infrared Spectra of copper thiol films a) full range b) narrower range, inset showing $-\mathrm{OH}$ region for $\mathrm{CsI}$ pellet CsI pellet (top line), diffuse reflectance of the same film (centre) and electrodeposited film (bottom). For this measurement a film prepared in methanol was used. 
The XPS and IR results together suggest the following reactions at the surface; competing oxide growth and conversion mean that the oxide thickness depends on a dynamic equilibrium between oxidation and reduction.

$$
\begin{aligned}
& \mathrm{O}_{2}+2 \mathrm{Cu} \rightarrow 2 \mathrm{CuO} \\
& 4 \mathrm{RSH}+2 \mathrm{CuO} \rightarrow \mathrm{RSSR}+2 \mathrm{RSCu}+2 \mathrm{H} 2 \mathrm{O}
\end{aligned}
$$

Supporting this, Sung et al[13] did not observe a stable thiolate layer with degassed ethanol on copper oxide surfaces, claiming only a monolayer to be present and full reduction to the metal. Keller[15] isolated the dithiol from a reaction between copper oxide and thiol.

Laibnis et al.[19] showed that thiols on copper usually have a layer of associated oxygen and $\mathrm{Cu}$ (I) at the interface even when steps are taken to minimize oxygen exposure and initial oxide thickness. Additionally they showed that the thiol layer becomes oxidized to the sulfonate slowly when exposed to the atmosphere (after removing it from the thiol solution). Laiho and Leiro[20] show that copper surfaces with initial oxide layers show higher thiol adsorption than a clean copper surface, they cite Himmelhaus et al[4] who suggest that there is a catalytic effect generating a denser monolayer. They also show that some oxide remains below the thiol film although most is reduced by the action of the thiol.

Keller et al[15] reported the formation of 20-90 nm thick layers of copper thiolate on copper powder in thiol solution. They hypothesized that an oxide layer was reduced to the copper (I) thiolate with the formation of the disulfide, evidenced by the molecular formula of the product and the presence of disulfide in solution after the reaction. This and the results presented here suggest that the increased thiol adhesion Laiho and Leiro[20] and Himmelhaus[4] observed was a multilayer of copper (I) thiolate formed from the pre-existing oxide.

Denayer et al[21 ] showed that varying the solvent affects the amount of oxide left at the interface and the density and purity of the layer. This follows an earlier study by Castner[22] showing an effect of the oxygen solubility in solvents at a gold interface.

Hutt and Liu[23] showed that a thiol layer on copper will withstand oxidation at low temperatures but at ambient temperatures an oxide layer formed over a few weeks, mostly comprised of $\mathrm{Cu} 2 \mathrm{O}$. In the measurements performed here the thiol solution remains active and so reduction of the newly formed oxide can continue, generating the copper (I) thiolate film.

We have observed oxide growth below thiol films and a small oxide contribution at the base of our thiolate layer. This can be explained by differences in the relative diffusion rates of oxygen and free thiol to the interface. Fondera et al.[24] showed that as the concentration of a thiol in solution is increased the thickness of the oxide film on copper is reduced and eventually fell below the threshold of their measurements. Although it has been suggested that the remaining oxygen on thiolated copper is due to a different type of oxide or hydroxide, this is not supported by our experiments, which instead indicate that a dynamic equilibrium of oxide growth and reduction is present but that the reduction stops at the copper (I) state, leaving a thick film of copper (I) thiolate.

\subsection{Amine Reagents}

In aminoethanol a thiol layer developed with the octanethiol that was much thinner than in any of the other solvents, it could not be imaged from the side in SEM as the others could. Small areas of nitrogen-containing material were left, as it was difficult to remove the aminoethanol from the surface.

Diethylenetriamine behaved similarly, in this case a misty film was observed at the surface of the material, but this was removed by water or ethanol rinsing. If it was retained, a high nitrogen and carbon EDX signal suggested that it consisted of a complex involving the solvent. This suggests that the formation of thiolate multilayers can be prevented by using amino-functionalised solvents. 
In butylamine again there was no evidence of thick film formation, EDX measurements showed mostly copper in the sampling volume along with some carbon. Oxygen levels were slightly lower than those on a freshly abraded sample, but it was not possible to tell from these measurements if the oxide layer at the interface between thiol and metal was thinner than samples prepared in ethanol. Samples standing in ethanol in the absence of thiol, however, darkened visibly and showed a measurable increase in oxygen by EDX over the same time. Investigation of the surface of the sample after 8 days in butlyamine/octanethiol revealed pitting, Figure $2 \mathrm{f}$ suggesting that corrosion continued on this sample too, but that the products were removed into solution.

Evaporation of the supernatant of the butylamine-thiol mixture or the alcohol-thiol mixtures after exposure to copper and oxygen left an oil; which is most likely to be the dioctyldisulfide, as found by Keller et. al.[15]

The supernatant of the amine/thiol mixtures after reaction with the copper samples was yellow to brown in color and turned light blue on exposure to water. Addition of copper acetate or copper oxide powder to a thiol/butylamine mixture produced a white suspension with a supernatant that turned dark blue on exposure to the air. Addition of copper acetate to an ethanol/thiol mixture produced a yellow-white precipitate that was stable to drying and had a similar infrared signature to the films, indicating that it is the copper(I) thiolate, in this case the solvent did not become colored during concentration or exposure to water. As amines are known to stabilize copper (I) in solution[25] it is likely that a $\mathrm{Cu}$ (I) complex involving the amine is formed, allowing copper (I) to be removed from the surface. Addition of water and oxygen would be expected to convert this to a blue copper (II) amine/water complex.

When a superhydrophobic copper thiolate grown in ethanol over 8 days was immersed in butylamine overnight the rough layer was removed and the butylamine became a green-blue color. This supports the argument that the Copper (I) complex is soluble in amines. As the color change did not appear in the samples corroding in butlyamine it is probable that copper oxidation is slower in the amine solutions than in alcohols, possibly due to removal of oxygen from the solution by copper catalyzed reaction with the thiol.[26] The brown colour of the amine solution after exposure to copper and thiol is likely to be due to the presence of $\mathrm{Cu}_{2} \mathrm{O}$ particles released during corrosion. These are incorporated into the films grown in other solvents.

\section{Conclusions}

Rough, superhydrophobic copper (I) thiolate films formed on copper in various alcohols including ethanol, which is the most common solvent used for thiol SAM formation. The rate of film growth and its morphology were dependent upon the alcohol used and its water content.

Although several days were required to form a superhydrophobic film the layer reached appreciable thicknesses over shorter periods and would form wherever the copper was exposed to oxygen and thiol at the same time. As the film is a copper thiolate it is difficult to distinguish a thin multilayer film from a SAM of the same thiol, so its presence may well account for the difficulties usually encountered in forming a clean monolayer at a copper surface.

Although we found copper oxidation in all of the solvents we used, thick thiolate films were not formed in amines, particularly butylamine, where material was removed into solution but a stable surface layer of bound thiol was still present.

Acknowledgements. We would like to acknowledge the EPSRC for funding and Emily Smith at Nottingham University and the Open Access scheme (EPSRC) for XPS access and characterization. 


\section{References}

1 Tarlov, M, US patent 5514501, 1996; Friebel, S., Aizenberg, J., Abad, S., Wiltzius, P., Appl. Phys. Lett. 77, 2406 (2000); Huang, J.; Hemminger, J. C. J. Am. Chem. Soc. 1993, 115, 3342;

2 Ishida, M., Kasuga, M., Kaneko, T., Shimoda, T., Jap. J. Appl. Phys. 2-Lett.,39: 3AB, (2000), L227-L229.

3 Tarlov, M. J., Burgess Jr., D. R. F., Gillen, G., J. Am. Chem. Soc., 115 :12, (1993), 5305-5306.

4 Himmelhaus, M., Gauss, I., Buck, M., Eisert, F., Wo“1l, Ch., Grunze, M., J. Electron Spectrosc. Relat. Phenom. 92 (1998) 139.

5 Sung, I. H., Kim, D. E., Tribology Lett., 17; 4, (2004), 835-844.

6 Chander, S. Kar, G., Fuerstenau, D. W., Corrosion Sci., 19: 6 (1979), 405-416.

7 Yamamoto, Y., Nishihara, H., Aramaki, K., J. Electrochem. Soc., 140: 2, (1993), 436-443.

8 Roach, P., Shirtcliffe, N. J., Newton, M. I., Soft Matter, 4: 2, (2008), 224-240. Shirtcliffe, N., McHale, G., Newton, M., J. Poly. Sci. 49; 17 (2011), 1203-1217.

9 Battino, R., Rettich, T., Tominaga, T., J. Phys. Chem. Ref. Data, 12: 2, (1983),163-178.

10 D'Souza, V., Iyer, V., Szmant, H., J. Org. Chem., 52; 9, (1987), 1720-1725.

11 Belmares, M., Blanco, M., Goddard III, W., Ross, R., Caldwell, G., Chou, S., Pham, J., Olofson, P., Thomas, C., J Comp. Chem., 25, 15. (2004), 1814-1826.

12 Adamson A and Gast, A., Physical Chemistry of Surfaces 6th ed., J. Wiley; New York, (1997).

13 Sung, M., Sung, K., Kim, C., Lee, S., Kim Y., J. Phys Chem B 104, (2000), 2273-2277.

14 MacIntyre, N., Cook, M., Anal. Chem., 47: 13, (1975), 2208-2213.; Thesis of D. Pop, TU Berlin available at TU Berlin website (http://www.diss.fu-berlin.de/diss/receive/FUDISS_thesis_000000001004)

15 Keller, H., Simaka, P., Schreppa W., Dembowski, J., Thin Solid Films, 244: 1-2, (1994), 799-805.

16 Wei, X., Fahlman, M., Epstein, A., Macromolecules, 32: 9, (1999), 3114-3117.

17 Briggs, D., Seah, M., (Editors), Practical Surface Analysis - Auger and X-ray Photoelectron Spectroscopy, Wiley Interscience; Chichester, (1990) (2nd ed. Vol. 1).

18 Papadimitropoulosa, G., Vourdasa, N., Vamvakasa, V., Davazoglou, D., Thin Solid Films, 515: 4, (2006), $2428-2432$.

19 Laibnis, P., Whitesides, G. Allara, D., Tao, Y., Parikh, A., Nuzzo, R., J. Am. Chem. Soc., 113, (1994), 7152-7167.

20 Laiho, T., Leiro, J., Appl. Surf. Sci., 252: 18, (2006), 6304-6312.

21 Denayer, J. Delhalle, J., Mekhalif, Z., Appl. Surf. Sci., 256, (2009), 1426-1430.

22 Castner, D., Langmuir, 12, (1996), 5083-5086.

23 Hutt, D., Liu, C., Appl. Surf. Sci. 252 (2005) 400-411.

24 Fonder, G., Laffineur, F., Delhalle J., Mekhalif, Z., J. Coll. Interf. Sci., 326: 2, 15, (2008), 333-338.

25 Kumbhara, A., Narasimhana S., Mathur, P., Analytica Chimica Acta, 294; 1, (1994), 103-111.

26 Smith, R., Reed, V., Hill, W., Phosphorus, Sulfur Silicon Rel. Elements90; 1-4, (1994), 147-154. 